\title{
The Application of Near Infrared Reflectance Spectroscopy as A Fast and Non-Destructive Method to Determine Inner Quality Parameters of Intact Mango
}

\author{
Agus Arip Munawar', Hesti Meilina ${ }^{2}$, Zulfahrizal $^{3}$ \\ ${ }^{1,3}$ Department of Agricultural Engineering, Syiah Kuala University, Banda Aceh, Indonesia \\ ${ }^{2}$ Department of Chemical Engineering, Syiah Kuala University, Banda Aceh, Indonesia \\ $\{1$ aamunawar@unsyiah.ac.id\}
}

\begin{abstract}
Generally speaking, to determine inner quality parameters such as soluble solids content (SSC) and vitamin C of intact mango or other fruits, several methods were already employed. Yet, most of them are based on chemical analysis and fruit extraction followed by other laboratory analysis. These methods often require complicated sample processing, longer time consuming and destructive. In the last three decades, the application of near infrared reflectance spectroscopy (NIRS) as a fast, robust and non-destructive method in agricultural industries is gaining more attentions. Thus, the main purpose of this present study is to apply the NIRS method in determining SSC and vitamin C of intact whole mango by developing prediction models. Near-infrared spectra data, in the form of diffuse reflectance spectrum, were acquired for a total of 53 mango samples. Spectra data were corrected and enhanced using mean normalization (MN), standard normal variate $(\mathrm{SNV})$ and the combination of them $(\mathrm{MN}+\mathrm{SNV})$. Prediction models used to predict SSC and vitamin C of intact mangos were developed using partial least square regression (PLSR). The results showed that SSC and vitamin $\mathrm{C}$ can be predicted rapidly and simultaneously using NIRS method with maximum correlation coefficient ( $\mathrm{r}$ ) were 0.85 for SSC and 0.96 for vitamin $\mathrm{C}$, with residual predictive deviation (RPD) index were 1.92 and 3.53 for SSC and vitamin C respectively. Based on obtained results, we may conclude that the NIRS method can be applied as an alternative fast and non-destructive method in determining quality parameters of intact mango.
\end{abstract}

Keywords: Soluble Solids Content (SSC), Near Infrared Reflectance Spectroscopy (NIRS), Mangos

\section{Introduction}

Mango, which is known as king fruit, is one of the most popular fruits around the world due to its taste, appearance and high nutritional value [1]. According to [2], approximately $50 \%$ of all tropical fruits produced worldwide are mangos. They are grown commercially in more than 87 countries with its production more than 43 million tons in 2017. As a country with a tropical climate, Indonesia contributes to worldwide total mango production with more than 673440 hectares, yielding $110902 \mathrm{Hg} / \mathrm{ha}$ in 2017 and is placed as the fourth of the twelve top mango producers in the world [3]. However, the total export of fresh fruit fluctuated in the last five years both in the total volume and the total value of major fresh fruit exports, whilst the fruit production remained relatively stable. One of the major problems currently restricting 
international trade in mangos is the strong heterogeneity in their physical maturity, which produces a low uniformity in ripening degree of fruit lots offered for sale [4].

In general, consumers purchase fresh fruits and vegetables on the basis of quality which is an important subject to those engaged in horticultural industries. Their acceptance depends on highly subjective factors including appearance, touch, smell, taste and even hearing. Soluble solids content (SSC) and vitamin C are two main quality parameters among others for mango fruit.

To determine these both quality parameters, several methods were employed. Yet, most of them were used based on solvent extraction with standard laboratory analysis. This analysis is sometimes complicated procedures, time-consuming and using of hazardous chemical solutions, from which may cause environmental pollutions. Therefore, unsuitable for the quality control of fresh agricultural products which requires real-time, rapid, on-line and non-destructive measurements [1].

Alternative fast, robust, non-destructive and pollution free methods are required to determine quality parameters of intact mango and other agricultural products. Near-infrared reflectance spectroscopy (NIRS) has been developed and widely applied in many sectors including in agriculture and horticulture industries [5]-[7]. The increasing importance of NIRS in agriculture is obvious from the recent increase in numbers of publications, as well as from the fact that many manufacturers and agricultural industries (e.g., grains, beverage, milk and dairy, and fruits and vegetables) have now implemented NIRS systems to measure and determine various quality parameters [8], [9].

Near-infrared reflectance spectroscopy (NIRS) technique works based on the principle of interaction of electromagnetic radiation with biological objects and thus it is considered to be suitable for determining the inner quality of food and agricultural product since this method is characterized by low labor costs, simple sample preparation, non-destructive, pollution free, and high speed of analysis. This technique gained widespread acceptance for analyzing agricultural products since its development in 1965 [8].

The overall research findings of our own studies show that NIRS was feasible to be applied as a rapid and non-destructive tool for quality attributes prediction. The prediction model performance was sufficiently robust and accurate with correlation coefficient (r) range of 0.87 -0.95 and residual predictive deviation (RPD) index was $1.14-2.15$ which is categorized as sufficient prediction models, and need some improvement.

Based on the advantages and excellence of NIRS as a novelty method to measure agricultural and food qualities, we attempted to apply the NIRS method in determining inner quality attributes of intact mango. So that it can be applied in sorting and grading processes. Thus, the main objective of this present study is to apply NIRS as a rapid, simultaneous and nondestructive method in quality parameters evaluation of intact mangos in form of SSC and vitamin $\mathrm{C}$ through prediction models.

\section{Methods}

\subsection{Samples}

A total of 58 intact mango samples were used in this experiment with different maturity stages from unripe to over-ripe stage. Samples were purchased in the local auction and stored in $25^{\circ} \mathrm{C}$ for two days to equilibrate before spectra acquisition and further chemical analysis. 


\subsection{Spectra data acquisition}

Near-infrared (NIR) spectra data of intact mango, samples were obtained using Fourier transform infrared instrument (FTIR, Thermo Nicolet Antaris II MDS). The basic measurement chosen for this spectra data measurement is high resolution with integrating sphere. Diffuse reflectance spectra data were acquired and recorded in the wavelength range from 1000 to 2500 $\mathrm{nm}$ with co-added 20 scans and averaged. Spectra data were recorded and saved in local computer with two different file formats (*.SPA and *.CSV)[1].

\subsection{Actual soluble solids content (SSC) and vitamin C measurements}

After acquiring and recording NIR spectra data, all mango samples were analyzed for their inner quality parameters in the form of soluble solids content (SSC) and vitamin C. Both these quality parameter measurements were carried out simultaneously by making juice from 25 grams of pulp sample and maximum of $100 \mathrm{ml}$ distilled water. A single drop filtered supernatant juice was squeezed and dropped onto a hand-held analog refractometer (model HRO32, Krüss Optronic $\mathrm{GmbH}$ ) to record SSC as oBrix [10] whilst automatic titration (model Titroline 96, Schott) with $0.1 \mathrm{~N} \mathrm{NaOH}$ to an end point of $\mathrm{pH} 8.1$ was used to measure vitamin $\mathrm{C}$ expressed as $\mathrm{mg} \cdot 100 \mathrm{~g}-1$ fresh mass [1]. All these two quality attributes were measured in triplicate and averaged.

\subsection{Spectra data correction}

Spectra data analysis was firstly performed to inspect spectra visualization and noise recognition. Noise, caused by light scattering were enhanced and corrected using mean normalization (MN), standard normal variate (SNV) and the combination of them (MN+SNV) methods.

\subsection{Prediction model}

Prediction models used to predict SSC and vitamin C of intact mangos, were developed simultaneously based on untreated raw and enhanced spectra data (MN, SNV, and MN+SNV). Prediction models were established using partial least square regression (PLSR) with 10 fold cross-validation [1].

\subsection{Prediction model performance}

Prediction models performances were judged for their accuracies and robustness using these following indicators: the coefficient of determination $\left(\mathrm{R}^{2}\right)$, correlation coefficient $(\mathrm{r})$, root mean square error (RMSE) and the residual predictive deviation (RPD) defined as the ratio between the standard deviation and the RMSE The higher the RPD, the greater and robust the model to predict SSC and vitamin C of intact mango samples [5]. It is obvious that the good model should have high $\mathrm{R}^{2}$ and $\mathrm{r}$ coefficient, low RMSE and few latent variables of PLSR [1]. 


\section{Result and Discussion}

\subsection{Spectra features of mango}

Typical diffuse near-infrared reflectance spectrum, after $\mathrm{MN}+\mathrm{SNV}$ correction for intact mango samples in the wavelength range from 1000 to $2500 \mathrm{~nm}$ is shown in Fig.1. this spectra data shows the absence of quality parameters of mango samples which are derived from the absorbance bands that result from the vibration of molecular bonds of O-H, C-H, C-O and N-H with the infrared radiation.

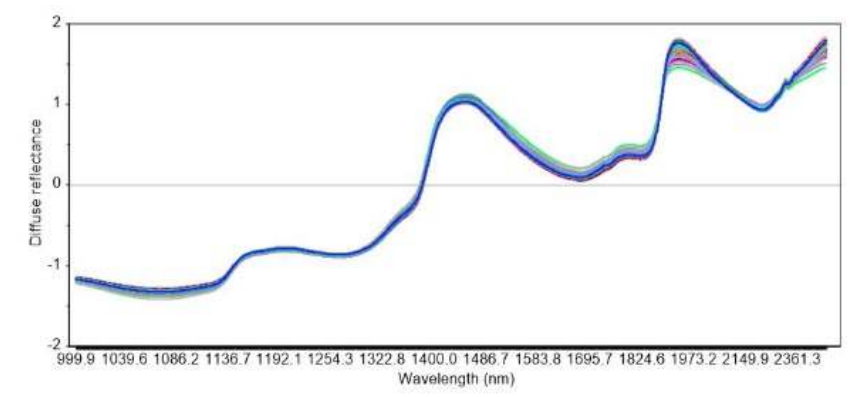

Fig.1. NIR spectra data of 58 intact mango samples after correction using MN+SNV method.

Intact mango samples were irradiated using infrared electromagnetic radiation and their reaction including reflectance, absorbance and transmittance are captured and recorded. The different reaction of this diffuse reflectance spectra data was depend on main chemical composition, cell structure and other chemical or physical properties of the samples. A captured near-infrared spectra of a biological object consists of the response of the molecular bonds O$\mathrm{H}, \mathrm{C}-\mathrm{H}, \mathrm{C}-\mathrm{O}$, and $\mathrm{N}-\mathrm{H}$. These bonds are subject to vibrational energy changes when irradiated by NIR frequencies [6]. The NIR spectrum indicates the presence of organic materials such as sugar content, vitamin, acidity, and other parameters.

As shown in Fig.1, spectra data were corrected and enhanced using a combination of mean normalization and standard normal variate $(\mathrm{MN}+\mathrm{SNV})$ method. Mean normalization were enhanced spectral data by normalizing spectra to its ideal condition which is normally mean spectra whilst SNV spectra corrected spectra data by scaling spectra to its suitable scale from 1 to $+1[1]$, [11].

It is obvious that mango contains more than $80 \%$ of water, the presence of water contents can be seen in Fig 1. where the highest peak was observed at wavelength $1490 \mathrm{~nm}$ and $1920 \mathrm{~nm}$ which were associated with $\mathrm{O}-\mathrm{H}$ bands. Furthermore, vitamin $\mathrm{C}$ which was constructed with $\mathrm{C}$ $\mathrm{H}-\mathrm{O}$ can be predicted in the wavelength range around $2100-2247 \mathrm{~nm}, 2312 \mathrm{~nm}$, and $2380 \mathrm{~nm}$.

\subsection{Prediction models}

The main part of near-infrared spectroscopy application is establishing calibration models used to predict quality attributes or chemical constituents of organic and biological samples. The most accurate and robust models were then transferred onto the NIR instrument for further quality evaluation. In this study, prediction models were developed using partial least square regression (PLSR). Prediction model consists of the relationship between the observed response variable y (Y-variables: SSC and Vitamin C) and the independent variable $\mathrm{x}$ (X-variables: NIR 
diffuse reflectance spectra data). The primary information that can be gathered from the interaction of the near-infrared radiation with the biological object is its physical, optical and chemical properties.

Calibration and prediction result for SSC and vitamin C of intact mango samples were shown in Table 1 and Table 2 respectively. Obtained results show that NIRS seems feasible to predict both main quality parameters of intact mangos (SSC and vitamin C) with sufficient performance for SSC $(\mathrm{RPD}=1.92$ and $\mathrm{r}=0.80)$ and excellent prediction performance for vitamin $\mathrm{C}(\mathrm{RPD}=$ 3.53 and $r=0.96)$. Their prediction performances were significantly improved when spectra data were corrected by MN and SNV methods as shown in both Tables.

Table 1. SSC prediction performance using diffuse reflectance spectra data

\begin{tabular}{ccccc}
\hline Spectrum & \multicolumn{4}{c}{ Statistical indicator } \\
& $\mathrm{R}^{2}$ & $\mathrm{r}$ & $\mathrm{RMSE}$ & $\mathrm{RPD}$ \\
\hline \hline Raw & 0.64 & 0.80 & 2.17 & 1.68 \\
$\mathrm{MN}$ & 0.66 & 0.81 & 2.10 & 1.74 \\
$\mathrm{SNV}$ & 0.72 & 0.85 & 1.90 & 1.92 \\
$\mathrm{MN}+\mathrm{SN}$ & 0.72 & 0.85 & 1.90 & 1.92 \\
$\mathrm{~V}$ & & & &
\end{tabular}

Table 2. Vitamin C prediction performance using diffuse reflectance spectra data

\begin{tabular}{ccccc}
\hline Spectrum & \multicolumn{4}{c}{ Statistical indicator } \\
& $\mathrm{R}^{2}$ & $\mathrm{r}$ & RMSE & $\begin{array}{c}\text { RP } \\
\mathrm{D}\end{array}$ \\
\hline \hline Raw & 0.63 & 0.81 & 4.34 & 1.65 \\
$\mathrm{MN}$ & 0.88 & 0.93 & 2.48 & 2.89 \\
$\mathrm{SNV}$ & 0.91 & 0.95 & 2.07 & 3.46 \\
$\mathrm{MN}+\mathrm{SN}$ & 0.92 & 0.96 & 2.03 & 3.53 \\
$\mathrm{~V}$ & & & & \\
\hline
\end{tabular}

Based on prediction results, both MN and SNV increased prediction accuracy and robustness. SNV seems provided more accurate than MN. The most accurate and robust prediction results were achieved when spectra data were enhanced and corrected using a combination of MN and $\mathrm{SNV}$ methods $(\mathrm{MN}+\mathrm{SNV})$. It provided maximum correlation coefficient $\mathrm{r}=0.85$ and $\mathrm{RPD}=$ 1.92 for $\mathrm{SSC}$ prediction and maximum $\mathrm{r}=0.96$ and $\mathrm{RPD}=3.53$ for vitamin $\mathrm{C}$ prediction. Scatter plot drawn for SSC prediction of intact mango samples was presented in Fig. 2. 

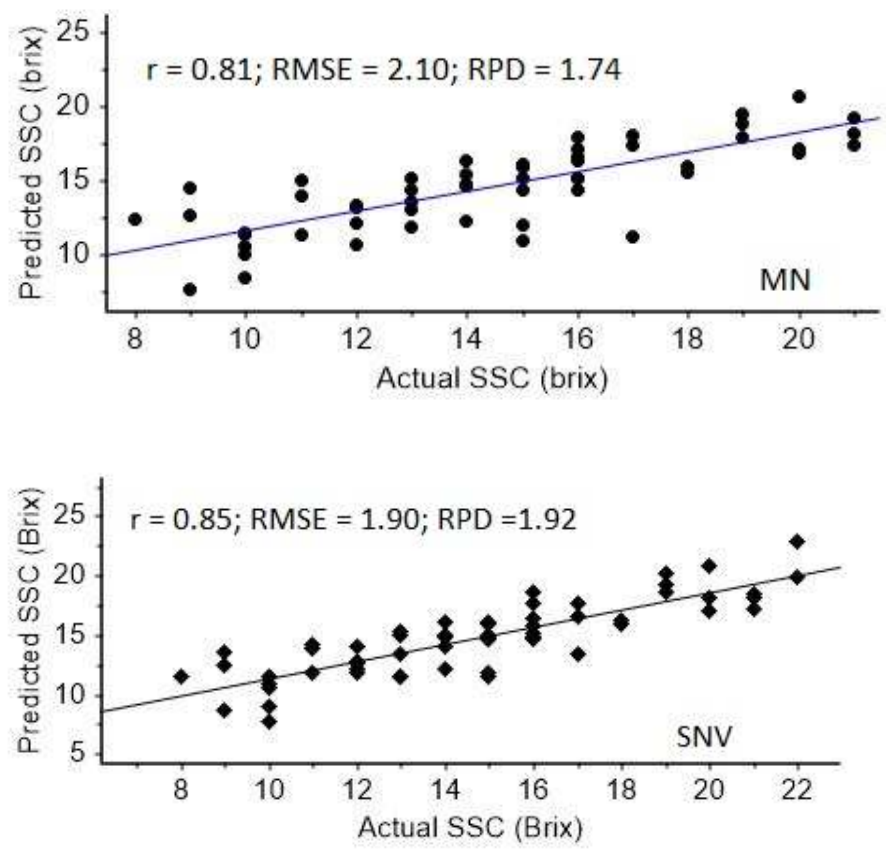

Fig. 2. Scatter plot between actual and predicted SSC of intact mangos using NIR spectra data.

In general, judging from prediction performance evaluation, NIRS can be used and improved by correcting and enhancing spectra data where in this study we use mean normalization (MN), standard normal variate (SNV) and combination of them (MN+SNV). The achieved RPD maximum was 1.92 which categorized as sufficient prediction performance. It can be enhanced and improved probably using a non-linear approach such as support vector regression (SVR).

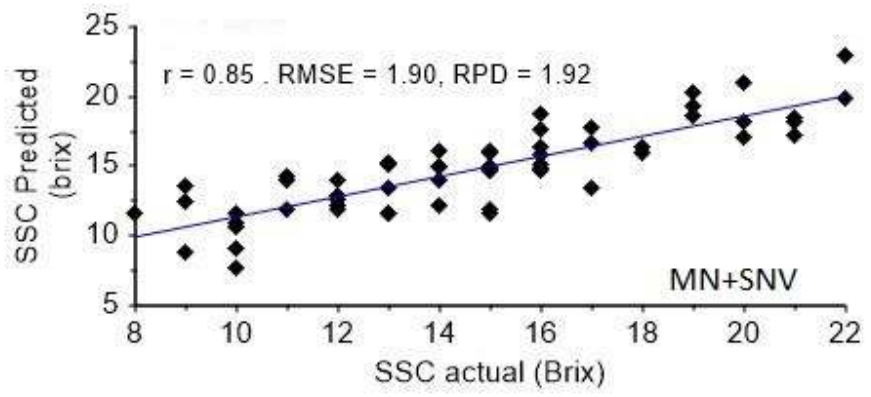

Moreover, excellent prediction results were achieved when NIRS used to predict vitamin C of intact mango samples. It reached maximum $\mathrm{r}$ coefficient of 0.96 using $\mathrm{MN}+\mathrm{SNV}$ spectrum. It improved from its initial prediction result using original un-treated spectrum where $r=0.81$. Scatter plot derived from actual and predicted vitamin C was presented in Fig.3. 

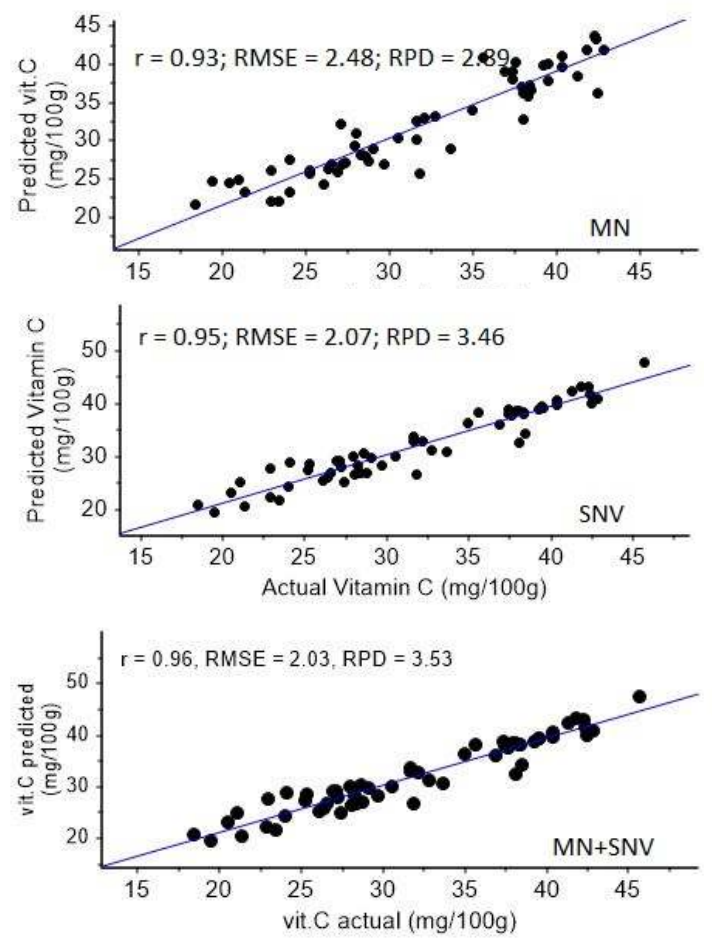

Fig. 3. Scatter plot between actual and predicted vitamin C of intact mangos using NIR spectra data

Based on regression curve derived from the best prediction models for SSC and vitamin C parameters, important and relevant wavelength for both main mango quality attributes were observed around $1418-1435 \mathrm{~nm}$, and between $2300-2415 \mathrm{~nm}$ as shown in Fig. 4.

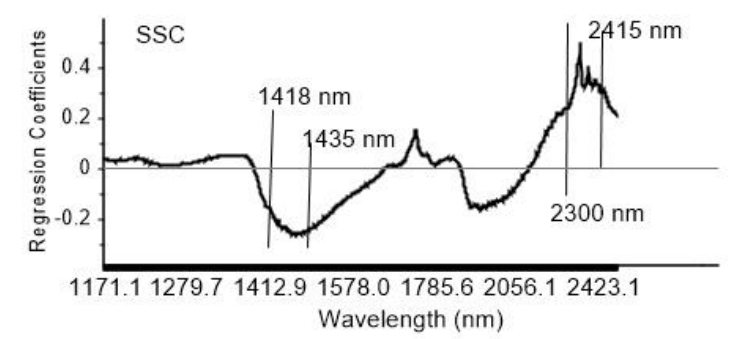

Fig. 3. Important wavelength in near-infrared region for SSC and Vitamin C prediction.

\section{Conclusion}

NIRS can be applied as an alternative fast, non-destructive and robust method used to predict two main quality parameters of intact mango namely soluble solids content (SSC) and Vitamin C. Spectra correction using mean normalization (MN) and standard normal variate (SNV) 
methods were significantly improved prediction accuracy and robustness. A combination of $\mathrm{MN}+\mathrm{SNV}$ spectra correction provide the most accurate and robust prediction result with correlation coefficient $r=0.85$ and $\mathrm{RPD}=1.92$ for SSC prediction, and maximum $\mathrm{r}=0.96$ and $\mathrm{RPD}=3.43$ for vitamin $\mathrm{C}$ prediction .

\section{Acknowledgments}

We sincerely acknowledge to DRPM, Ministry of Research, Technology and Higher Education for providing research grant and funding through PKLN scheme 2017/2018.

\section{REFERENCES}

[1] A. A. Munawar, D. von Hörsten, J. K. Wegener, E. Pawelzik, and D. Mörlein, "Rapid and non-destructive prediction of mango quality attributes using Fourier transform nearinfrared spectroscopy and chemometrics," Eng. Agric. Environ. Food, vol. 9, no. 3, pp. 208-215, 2016.

[2] S. Jedele, A. M. Hau, and M. von Oppen, "An analysis of the world market for mangos and its importance for developing countries," in Conference on International Agricultural Research for Development, 2003, pp. 1-11.

[3] FAO, "Mangos production and yield in 2017," FAO, 2008. .

[4] L. Chen, X. Xue, Z. Ye, J. Zhou, F. Chen, and J. Zhao, "Determination of Chinese honey adulterated with high fructose corn syrup by near infrared spectroscopy," Food Chem., vol. 128, no. 4, pp. 1110-1114, 2011.

[5] P. Jaiswal, S. N. Jha, and R. Bharadwaj, "Non-destructive prediction of quality of intact banana using spectroscopy," Sci. Hortic. (Amsterdam)., vol. 135, pp. 14-22, 2012.

[6] E. Bobelyn, A.-S. Serban, M. Nicu, J. Lammertyn, B. M. Nicolai, and W. Saeys, "Postharvest quality of apple predicted by NIR-spectroscopy: Study of the effect of biological variability on spectra and model performance," Postharvest Biol. Technol., vol. 55, no. 3, pp. 133-143, 2010.

[7] P. Penchaiya, E. Bobelyn, B. E. Verlinden, B. M. Nicolaï, and W. Saeys, "Nondestructive measurement of firmness and soluble solids content in bell pepper using NIR spectroscopy," J. Food Eng., vol. 94, no. 3, pp. 267-273, 2009.

[8] B. M. Nicolaï et al., "Nondestructive measurement of fruit and vegetable quality by means of NIR spectroscopy: A review," Postharvest Biol. Technol., vol. 46, no. 2, pp. 99-118, 2007.

[9] D. Cozzolino, W. U. Cynkar, N. Shah, and P. Smith, "Multivariate data analysis applied to spectroscopy: Potential application to juice and fruit quality," Food Res. Int., vol. 44, no. 7, pp. 1888-1896, 2011.

[10] Z. Xiaobo, Z. Jiewen, H. Xingyi, and L. Yanxiao, Use of FT-NIR spectrometry in noninvasive measurements of soluble solid contents (SSC) of 'Fuji' apple based on different PLS models, vol. 87. 2007.

[11] Samadi, S. Wajizah, and A. A. Munawar, "Fast and simultaneous prediction of animal feed nutritive values using near infrared reflectance spectroscopy Fast and simultaneous prediction of animal feed nutritive values using near infrared reflectance spectroscopy," in International Conference on Agriculture, Environment, and Food Security, 2018. 